Ecología

\title{
Escarabajos atraídos a la carroña (Coleoptera: Scarabaeidae, Geotrupidae, Hybosoridae, Trogidae y Silphidae) en las cañadas de Coatepec Harinas, Estado de México, México
}

\author{
Beetles attracted to carrion (Coleoptera: Scarabaeidae, Geotrupidae, Hybosoridae, Trogidae \\ and Silphidae) in the glens of Coatepec Harinas, Estado de México, Mexico \\ Juan Carlos Pérez-Villamares ${ }^{\mathrm{a}}$, Esteban Jiménez-Sánchez ${ }^{\mathrm{b}, *}$ y Jorge Padilla-Ramírez ${ }^{\mathrm{b}}$ \\ ${ }^{\text {a }}$ Facultad de Ciencias, Universidad Autónoma del Estado de México, 50000, Instituto Literario 100, Col. Centro, Toluca, Estado de México, México \\ ${ }^{\mathrm{b}}$ Facultad de Estudios Superiores Iztacala, Universidad Nacional Autónoma de México, Laboratorio de Zoología, Av. de los Barrios 1, Los Reyes Iztacala, 54090, \\ Tlalnepantla, Estado de México, México
}

Recibido el 1 de junio de 2015; aceptado el 11 de diciembre de 2015

Disponible en Internet el 19 de abril de 2016

\begin{abstract}
Resumen
Las cañadas de Coatepec Harinas, ubicadas al sur del Nevado de Toluca, son de interés para la conservación por su integridad biológica. En este estudio se presenta la composición de Scarabaeidae, Geotrupidae, Hybosoridae, Trogidae y Silphidae, además se analizan aspectos como abundancia, riqueza, diversidad y estacionalidad. Esta información servirá para el desarrollo de estudios comparativos posteriores realizados fuera de las cañadas. Los muestreos fueron mensuales durante un año con trampas tipo NTP-80 cebadas con calamar, en un bosque de encino con elementos de mesófilo de montaña. Se obtuvieron 1,513 individuos pertenecientes a 9 especies y 8 géneros. Silphidae (94.3\%) fue la familia más abundante, seguida por Scarabaeidae (4.7\%), ambas con 3 especies cada una; las restantes, una especie y menos de 10 ejemplares. Nicrophorus mexicanus Matthews, 1888 (Silphidae), Nicrophorus olidus Matthews, 1888 (Silphidae), Oxelytrum discicolle (Brullè, 1840) (Silphidae) y Oniticellus rhinocerulus Bates, 1889 (Scarabaeidae) agruparon el 99\%. Germarostes Paulian, 1982 (Hybosoridae) se registró por primera vez para el Estado de México. La estacionalidad fue marcada: el $\mathbf{8 7 . 5 \%}$ de la abundancia y las 9 especies obtenidas se registraron en las lluvias. La diversidad y la cobertura de la vegetación, así como la presencia de arroyos, propiciaron condiciones favorables para las 4 especies más abundantes.

Derechos Reservados (C) 2016 Universidad Nacional Autónoma de México, Instituto de Biología. Este es un artículo de acceso abierto distribuido bajo los términos de la Licencia Creative Commons CC BY-NC-ND 4.0.
\end{abstract}

Palabras clave: NTP-80; Bosque mesófilo de montaña; Bosque de encino; Gremios tróficos; Nicrophorus; Oniticellus

\section{Abstract}

The glens of Coatepec Harinas in the southern part of Nevado de Toluca are of conservation concern because of their biological integrity. This study reveals the composition of the Scarabaeidae, Geotrupidae, Hybosoridae, Trogidae and Silphidae of the Coatepec Harinas' glens, and analyzes their abundance, species richness, diversity and seasonality. Such information can be used for further comparative studies conducted outside the glens. Monthly samples were obtained during a year using traps type NTP-80 baited with squid in an oak forest with some elements of cloud forest. A total amount of 1,513 individuals belonging to 9 species and 8 genera were collected. Silphidae (94.3\%) was the most abundant family, followed by Scarabaeidae (4.7\%), both of which had 3 species each; the remaining families had 1 species and less than 10 specimens.

\footnotetext{
* Autor para correspondencia.

Correo electrónico: estjimsan@yahoo.com.mx (E. Jiménez-Sánchez).

La revisión por pares es responsabilidad de la Universidad Nacional Autónoma de México.
} 
Moreover, Nicrophorus mexicanus Matthews, 1888 (Silphidae), Nicrophorus olidus Matthews, 1888 (Silphidae), Oxelytrum discicolle (Brullè, 1840) (Silphidae) and Oniticellus rhinocerulus Bates, 1889 (Scarabaeidae) grouped together 99\% of individuals. Germarostes Paulian, 1982 (Hybosoridae) was recorded for the first time in the Estado de México. The seasonality was evident, $87.5 \%$ of the abundance and the 9 species were collected during the rainy season. The diversity of plants and tree canopy cover, as well as the presence of the stream, propitiated favorable conditions for the 4 most abundant species.

All Rights Reserved (C) 2016 Universidad Nacional Autónoma de México, Instituto de Biología. This is an open access item distributed under the Creative Commons CC License BY-NC-ND 4.0.

Keywords: NTP-80; Cloud forest; Oak forest; Trophic guilds; Nicrophorus; Oniticellus

\section{Introducción}

El sistema de cañadas situadas al sur del Nevado de Toluca en el municipio de Coatepec Harinas, en el centro de México, alberga fragmentos de bosque mesófilo de montaña en sus laderas por debajo de los 2,700 m de altitud (Torres-Zúñiga y Tejero-Diez, 1998), comunidades que son de las más ricas en cuanto a diversidad de especies y con un porcentaje elevado de endemismos (Toledo, 2009), las cuales en esta región se encuentran muy amenazadas por el avance de la floricultura y de varios tipos de cultivos (Ceballos, 2011). Debido a que la zona aún conserva una integridad biológica significativa con oportunidad real de conservación, la Comisión Nacional para el Conocimiento y Uso de la Biodiversidad (Conabio) la ha incluido dentro de la Región Terrestre Prioritaria 109 «Nevado de Toluca» (Arriaga et al., 2000), dentro de la cual también queda incluida el Área de Protección de la Flora y la Fauna Nevado de Toluca, que abarca tipos de vegetación presentes por arriba de los 3,000 m de altura como son el zacatonal alpino, el bosque de pino y el bosque mixto de pino-encino; este último es la continuación con el bosque mesófilo de montaña de las zonas más bajas de las cañadas (Conanp, 2013). Sin embargo, hace falta conocer con precisión la flora y la fauna que habita en estos lugares, lo cual ayudará en la toma de decisiones sobre conservación. Además, se ha visto que las cañadas y los bosques menos fragmentados constituyen lugares muy importantes para el establecimiento de las poblaciones de escarabajos especializados en el aprovechamiento de la carroña y el excremento (Caballero y León-Cortés, 2012).

En particular el conocimiento de los coleópteros necrófilos en el Estado de México es muy escaso para la mayoría de sus regiones desde el punto de vista del inventario de las especies (Jiménez-Sánchez, Labrador, López, Navarrete-Heredia y Padilla, 2009). Para las familias abordadas en esta investigación, en el Estado se tienen los estudios realizados por CejudoEspinosa y Deloya (2005), Méndez (2002), Morón y Zaragoza (1976) y Trevilla-Rebollar, Deloya y Padilla-Ramírez (2010); la mayoría de estos han sido enfocados en los escarabajos asociados a la carroña, que constituyen un gremio importante desde el punto de vista ecológico por su participación como degradadores de la materia orgánica de origen animal.

El presente estudio tiene como objetivo describir la composición de la fauna de coleópteros Scarabaeidae, Geotrupidae, Hybosoridae, Trogidae y Silphidae atraídos a trampas tipo NTP80 en las cañadas de Coatepec Harinas, Estado de México, México, así como analizar aspectos ecológicos, por ejemplo la abundancia, la riqueza, la diversidad y la estacionalidad. El análisis de estos parámetros ecológicos y los patrones observados en hábitats relativamente sin impacto antropogénico, como el caso del área de estudio, puede brindar información para el desarrollo de estudios comparativos posteriores llevados a cabo fuera de las cañadas, lo cual permitirá el monitoreo ambiental y el manejo en la conservación.

\section{Materiales y métodos}

El municipio de Coatepec Harinas, Estado de México, se localiza en el centro del país, al sur del Nevado de Toluca. Forma parte de la Región Hidrológica del Balsas, específicamente a la cuenca del río Amacuzac. La región está constituida por una serie de cañadas y tiene una altitud promedio de 2,270 m. Presenta un clima templado subhúmedo (Cw) (García, 1973), con una precipitación total anual de 1,130 mm. El periodo de lluvias va de mayo a octubre, con precipitaciones medias mensuales mayores a $80 \mathrm{~mm}$ por mes, y el de sequía de noviembre a abril, con registros menores de $35 \mathrm{~mm}$. La temperatura media anual es de $16^{\circ} \mathrm{C}$ (Conagua, 2011).

\section{Sitio de muestreo}

Se ubicó entre los $18^{\circ} 56^{\prime} 9.5^{\prime \prime} \mathrm{N}$ y $\operatorname{los} 99^{\circ} 45^{\prime} 35.9^{\prime} \mathrm{O}$, a una altitud promedio de 2,234 m en una cañada del área. La vegetación correspondió a los bosques de encino y mesófilo de montaña distribuidos en las orillas de las cañadas. En la parte más expuesta de estas se desarrollan encinares de Quercus laeta Liemb. 1854 , Q.mexicana Bonpl. 1809, Q.obtusata Humb. y Bonpl. 1809 y Q.crassipes Humb. y Bonpl. 1809. En las áreas con menor insolación y elevada humedad se encuentran encinares y bosque mesófilo de montaña, donde dominan Q. candicans Née 1801, Q. castanea Née 1801 y Q. laurina Humb. y Bonpl. 1809. Dentro de esta última vegetación, cerca de los arroyos y cañadas, los encinos expuestos se acompañan de un conjunto de árboles típicamente higrófilos como Alnus acuminata Kunth 1917 (ripícola), Buddleja cordata H.B.K., Carpinus caroliniana Walter, Clethra mexicana DC., Cleyera mexicana (Turcz.), C. disciflora DC., Fraxinus uhdei (Wenz.) Lingelsh. (1907), Garrya laurifolia (Benth.) Hartw. Ilex tolucana Hemsl., Meliosma dentata (Liebm.) Urb., Oreopanax xalapensis (Kunth) Decne y Planc., Prunus prionophylla Standley 1919, Pr. serotina Ehrenb., Rapanea jurgensenii Mez., Symplocos citrea Lex., Ternstroemia 
lineata DC., Tilia mexicana Schlechtendal, Xylosma flexuosa (Kunth) Hemsl. y Zinowewia concinna Lundell. Generalmente forman parte de estos bosques una gran diversidad de especies epífitas de las familias Bromeliaceae, Orchidaceae, Polypodiaceae y Piperaceae. El sotobosque a su vez es rico en especies, e incluso con coberturas del 30 al $50 \%$, donde dominan especies de Adiantum, Archibaccharis, Bidens, Cestrum, Dryopteris, Eupatorium, Iresine, Lippia, Montanoa y Salvia (Ceballos, 2011).

\section{Muestreo}

Los muestreos fueron mensuales de agosto de 2010 a julio de 2011. Se colocaron 2 transectos con una separación entre estos de aproximadamente $1 \mathrm{~km}$, cada uno con 4 trampas permanentes tipo NTP-80 (Morón y Terrón, 1984), sin hacer distinción entre las asociaciones vegetales descritas. Para abarcar la heterogeneidad del hábitat las trampas fueron distribuidas desde la parte superior de la cañada $-2,540 \mathrm{~m}$ snm—, que correspondió a la zona expuesta, hasta el fondo de la misma - 2,210 $\mathrm{m} \mathrm{snm,-}$ donde hubo mayor cobertura vegetal cerca de un arroyo de corriente continua. Entre una y otra trampa hubo una diferencia de aproximadamente $50 \mathrm{~m}$ de altitud. Cada trampa fue cebada con calamar, y como líquido conservador se utilizó una mezcla de 95 partes de alcohol al $70 \%$ y 5 partes de ácido acético glacial; tanto el cebo como el líquido fueron sustituidos mensualmente y el material biológico recuperado se colocó en frascos con alcohol al 70\% para su trasladado al laboratorio. Los ejemplares se depositaron en la Colección de Artrópodos de la Facultad de Estudios Superiores Iztacala (CAFESI), Universidad Nacional Autónoma de México.

Para el arreglo de la categoría a nivel de familia se siguió el criterio taxonómico de Bouchard et al. (2011). Para la identificación de los Silphidae se usaron las claves de Navarrete-Heredia (2009), mientras que para los pertenecientes a Scarabaeidae, Hybosoridae y Geotrupidae se emplearon los trabajos de Bitar y Delgado (2009), Deloya y Morón (1994), Delgado, Pérez y Blackaller (2000), Halffter y Martínez (1962), Morón y Zaragoza (1976) y Zunino y Halffter (1988). Para Trogidae se utilizaron las claves de Vaurie (1955) y la identificación fue corroborada por el Dr. Cuauhtémoc Deloya, del Instituto de Ecología, A.C. Xalapa, Veracruz, especialista del grupo.

\section{Análisis de los datos}

El análisis ecológico incluyó el número de especies —riqueza—, el número de organismos —abundancia- y la presencia de los adultos a lo largo del año. También se calculó la uniformidad y el índice de diversidad de Shannon para cada época del año; la diversidad entre estas se comparó mediante una prueba de $t$ de Hutcheson (Magurran, 1989). Los cálculos fueron hechos con el programa Past, versión 2.12 (Hammer, Harper y Ryan, 2001). Con el objetivo de conocer si existía correlación de la riqueza y la abundancia mensual con la precipitación, se realizó la prueba de Spearman debido a que los datos no mostraron una distribución normal (Zar, 1999).

\section{Resultados}

Se obtuvieron un total de 1,513 coleópteros. La familia más abundante fue Silphidae, con 1,427 individuos (94.3\%), seguida por Scarabaeidae con 71 (4.7\%), Hybosoridae con 10 (0.6\%), Geotrupidae con $4(0.26 \%)$ y Trogidae con uno (tabla 1$)$. Se registraron un total de 9 especies representantes de 8 géneros. Todos los ejemplares fueron determinados a nivel de especie excepto los de Hybosoridae, que pertenecieron a Germarostes, género que se registra por primera vez para el Estado de México. Scarabaeidae y Silphidae presentaron la mayor riqueza de especies con 3 cada una, incluidas en 3 y 2 géneros, respectivamente. Hybosoridae, Geotrupidae y Trogidae tuvieron la menor riqueza, con una especie cada una (tabla 1). Cuatro especies agruparon el $99 \%$ de la abundancia total, de las cuales 3 correspondieron a Silphidae: Nicrophorus mexicanus Matthews, 1888 fue la dominante, con el 55\% de los individuos, seguida por Nicrophorus olidus Matthews, 1888, con el 33\%, y Oxelytrum discicolle (Brullè, 1840), con el 6.3\%; la cuarta fue el Scarabaeidae Oniticellus rhinocerulus Bates, 1889, con el 4.4\%; las 5 restantes tuvieron menos de 11 individuos (1\%) (tabla 1).

Los coleópteros necrófilos adultos tuvieron una estacionalidad marcada: el $87.5 \%$ de la abundancia fue capturada durante la temporada de lluvias y el restante $12.5 \%$, en la sequía. Durante el periodo húmedo la mayor abundancia fue en junio, con $34 \%$; de enero a marzo se obtuvieron los valores más bajos de abundancia, con menos de 10 individuos (fig. 1A). La precipitación tuvo una correlación positiva con la riqueza (correlación de Spearman, $\left.r^{2}=0.779, p<0.05\right)$ y con la abundancia $\left(r^{2}=0.777\right.$, $p<0.05)$. Nueve especies se registraron en las lluvias, de las cuales 5 fueron exclusivas de este periodo. En la sequía se obtuvieron solo 4 , y ninguna de ellas fue exclusiva. La diversidad en el periodo de las lluvias $\left(\mathrm{H}^{\prime}=1.09\right)$ fue significativamente mayor que la obtenida en la sequía $\left(H^{\prime}=0.87\right)$ (prueba de $t$, $\mathrm{gl}=244.1, p=0,001)$. En el periodo de lluvias se registraron de 3 a 7 especies por mes y un total de 9 en esta época, de las cuales 7 coexistieron en julio. En la sequía se obtuvieron de 1 a 4 especies por mes (fig. 1B), y estas correspondieron a las 3 especies de Silphidae y una de Scarabaeidae (O. rhinocerulus); las 4 coexistieron en noviembre y tuvieron una correlación positiva con la precipitación media mensual (prueba de Spearman, $N$. mexicanus, $r^{2}=0.860, p<0.05 ; N$. olidus, $r^{2}=0.640, p<0.05$ y O.rhinocerulus, $r^{2}=0.632, p<0.05$ ), excepto $O$. discicolle $\left(r^{2}=0.261, p=0.411\right)$.

De enero a marzo solo estuvo presente $N$. mexicanus y fue la única especie anual, por lo que la familia Silphidae se mantuvo activa durante todo el año. En el caso de N.olidus fue casi exclusiva de la época de lluvias, solo estuvo ausente de enero a marzo. En cuanto al número de individuos, N. olidus superó a las poblaciones de $N$. mexicanus en mayo y junio, mientras que la segunda fue más abundante de julio a diciembre, notándose un claro desfase de sus picos máximos de abundancia (fig. 2). La menos abundante de las 3 especies de Silphidae fue $O$. discicolle, la cual apareció de mayo a julio, periodo que coincidió con la época de lluvias; también se recolectó de octubre a diciembre, que correspondió con el inicio de la sequía (tabla 1). 
Tabla 1

Abundancia, riqueza absoluta y acumulada por mes, de los coleópteros atraídos a NTP-80 de Coatepec Harinas, Estado de México.

\begin{tabular}{|c|c|c|c|c|c|c|c|c|c|c|c|c|c|}
\hline & AGO & SEP & OCT & $\mathrm{NOV}$ & DIC & ENE & FEB & MAR & $\mathrm{ABR}$ & MAY & JUN & JUL & Total \\
\hline Silphidae & & & & & & & & & & & & & 1,427 \\
\hline \multicolumn{14}{|l|}{ Nicrophorinae } \\
\hline Nicrophorus mexicanus & 143 & 79 & 21 & 83 & 17 & 1 & 9 & 5 & 19 & 112 & 189 & 154 & 832 \\
\hline Nicrophorus olidus & 6 & 3 & 1 & 21 & 5 & - & - & - & 8 & 147 & 270 & 39 & 500 \\
\hline \multicolumn{14}{|l|}{ Silphinae } \\
\hline Oxelytrum discicolle & - & - & 1 & 5 & 5 & - & - & - & - & 40 & 42 & 2 & 95 \\
\hline \multicolumn{14}{|l|}{ Scarabaeidae } \\
\hline \multicolumn{14}{|l|}{ Scarabaeinae } \\
\hline Oniticellus rhinocerulus & 3 & 10 & 1 & 11 & - & - & - & - & - & - & 3 & 39 & 67 \\
\hline Onthophagus chevrolati & - & - & - & - & - & - & - & - & - & - & 1 & 1 & 2 \\
\hline \multicolumn{14}{|l|}{ Dynastinae } \\
\hline Xyloryctes thestalus & - & - & - & - & - & - & - & - & - & - & - & 2 & 2 \\
\hline \multicolumn{14}{|l|}{ Hybosoridae } \\
\hline Germarostes sp. & 2 & 2 & - & - & - & - & - & - & - & - & - & 6 & 10 \\
\hline \multicolumn{14}{|l|}{ Geotrupidae } \\
\hline Ceratotrupes bolivari & - & - & - & - & - & - & - & - & - & - & 4 & - & 4 \\
\hline \multicolumn{14}{|l|}{ Trogidae } \\
\hline Trox plicatus & - & 1 & - & - & - & - & - & - & - & - & - & - & 1 \\
\hline Abundancia & 154 & 95 & 24 & 120 & 27 & 1 & 9 & 5 & 27 & 299 & 509 & 243 & 1,513 \\
\hline Riqueza & 4 & 5 & 4 & 4 & 3 & 1 & 1 & 1 & 2 & 3 & 6 & 7 & 9 \\
\hline Riqueza acumulada & 4 & 5 & 6 & 6 & 6 & 6 & 6 & 6 & 6 & 6 & 8 & 9 & 9 \\
\hline
\end{tabular}

Los meses sombreados en gris corresponden al periodo de lluvias.
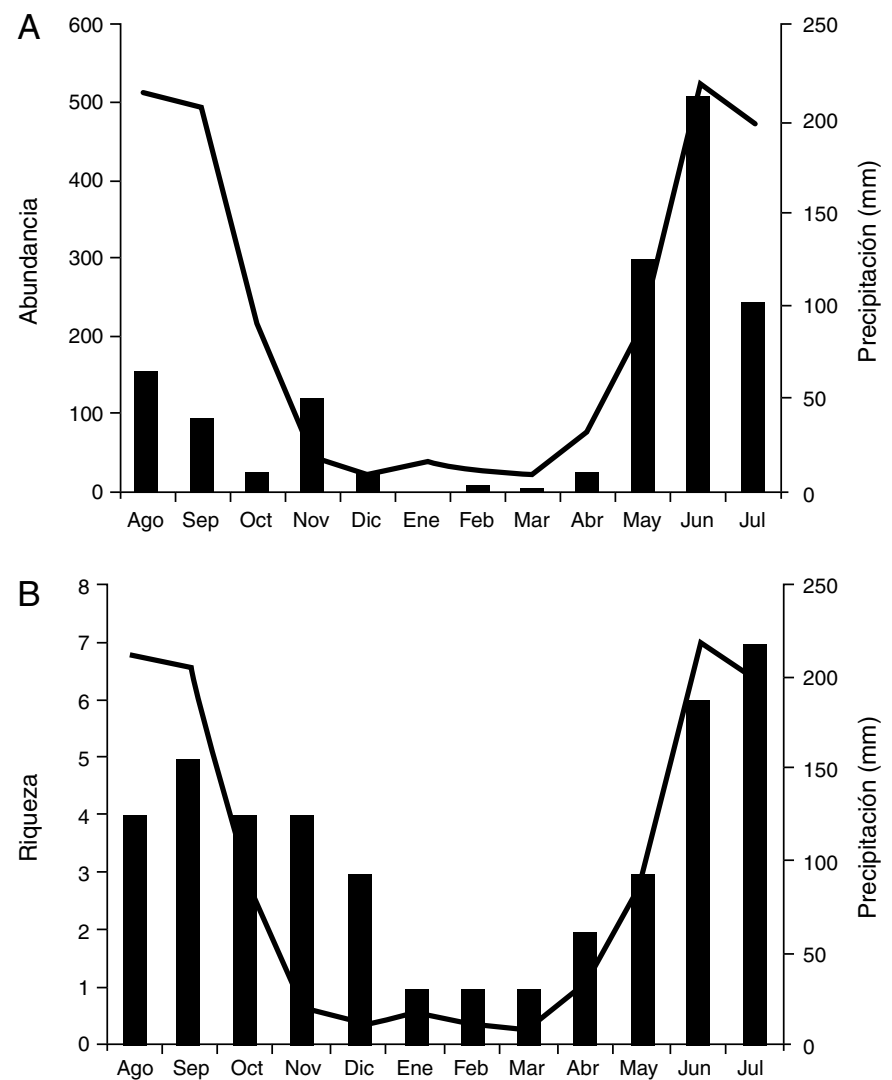

Figura 1. Fluctuación anual de la abundancia (A) y la riqueza (B) (barras negras) de los coleópteros atraídos a NTP-80 y su relación con la precipitación (línea), en Coatepec Harinas, Estado de México.

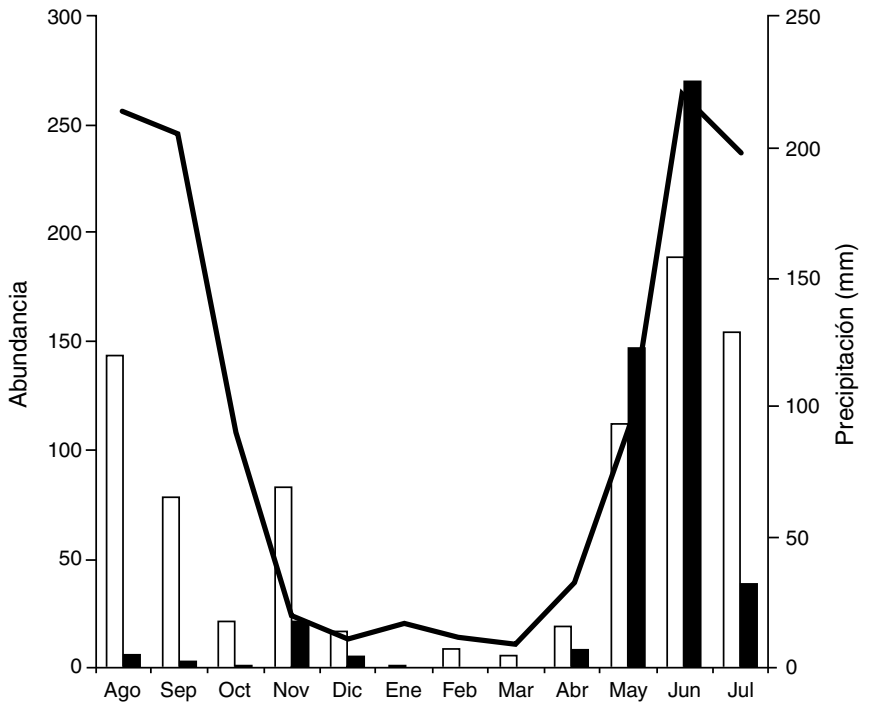

Figura 2. Fluctuación anual de la abundancia de Nicrophorus mexicanus (barra blanca) y Nicrophorus olidus (barra negra) y su relación con la precipitación (línea), en Coatepec Harinas, Estado de México.

Scarabaeidae se presentó exclusivamente en las lluvias y el primer mes de sequía; sus 3 especies se registraron en julio, y el resto del tiempo se encontró de 1 a 2 especies. Dicha estacionalidad fue muy evidente para O.rhinocerulus, que fue la especie dominante de las 3 obtenidas para esta familia, mientras que Onthophagus chevrolati chevrolati Harold, 1869 y Xyloryctes thestalus Bates, 1888, se presentaron esporádicamente. Hybosoridae, Geotrupidae y Trogidae solo estuvieron 
en la época de lluvias; la primera, representada por Germarostes, se observó de julio a septiembre; la segunda, que incluyó a Ceratotrupes bolivari (Halffter y Martínez, 1962), únicamente se capturó en junio, y Trox plicatus Robinson, 1940, solo se capturó en septiembre (tabla 1). De acuerdo con los grupos funcionales propuestos por Deloya, Parra-Tabla y Delfín-González (2007), 7 especies quedaron incluidas en el grupo funcional saprófago y 2 en el saprofitófago; al primero de estos correspondieron las especies de los siguientes gremios: 1) necrófagas: $N$. mexicanus, $N$. olidus y $O$. discicolle; 2) coprófagas: C. bolivari, O.rhinocerulus y O.c. chevrolati, y 3) telionecrófago: T.plicatus. Dentro de las saprofitófagas se incluyó a X.thestalus y Germarostes sp. Sin embargo, el gremio no quedó definido debido a que se desconoce el hábito alimentario del adulto.

\section{Discusión}

La dominancia de Silphidae sobre el resto de las familias capturadas en las cañadas de Coatepec Harinas, Estado de México, se debe a que sus especies están mejor adaptadas a comunidades montanas donde se registran sus mayores abundancias (Peck y Anderson, 1985). Los sílfidos N.mexicanus, N.olidus y O.discicolle obtenidos en el área de estudio son de amplia distribución en México, y es común encontrarlos en simpatría en sitios con bosques de pino, encino, pino-encino y mesófilo de montaña, por debajo o alrededor de los 2,000 m, como fue el caso de Coatepec Harinas (Halffter, Favila y Arellano, 1995; Navarrete-Heredia, 2009; Navarrete-Heredia y Quiroz-Rocha, 2000; Naranjo-López y Navarrete-Heredia, 2011; Quiroz-Rocha, Navarrete-Heredia y Martínez, 2008; Rivera-Cervantes y García-Real, 1998; Trevilla-Rebollar et al., 2010). Por el contrario, en tierras bajas con bosque tropical caducifolio (900 m) (Gómez, 2005) y matorral xerófilo (1,400 m) (Jiménez-Sánchez, Quezada-García y Padilla-Ramírez, 2013) se han encontrado 1 o 2 especies con poca abundancia. Lo anterior coincide con lo observado a lo largo de un transecto altitudinal realizado en la Zona de Transición Mexicana, donde solo se capturaron 2 especies entre 1,100 y $1,530 \mathrm{~m}$, mientras que entre 1,770 y 2,000 m se registró el mayor número de especies, con 4, y entre 2,340 y $2,600 \mathrm{~m}$ se obtuvo la mayor abundancia de Silphidae (Halffter et al., 1995). También, la riqueza se incrementa cuando en las zonas aledañas se desarrollan otros tipos de vegetación y los sitios constituyen áreas de solapamiento de la fauna, como lo ocurrido en algunas localidades de bosque tropical caducifolio, donde se han registrado de 3 a 4 especies (Méndez, 2002; Naranjo-López y Navarrete-Heredia, 2011; Reyes, 2001).

La estacionalidad fue muy marcada para todas las familias, lo cual es un patrón común para estos grupos. Caso especial fue $N$.mexicanus, que se registró todo el año. Este hecho se ha repetido en otros estudios (Arellano, Favila y Huerta, 2005; Rivera-Cervantes y García-Real, 1998; Terrón, Anduaga y Morón, 1991; Zaragoza y Pérez, 1975), lo mismo que el predominio sobre N.olidus por arriba de $\operatorname{los} 2,000 \mathrm{~m}$ de altura (Navarrete-Heredia, 1995; Rivera-Cervantes y García-Real, 1998). Por su parte, N. olidus mostró una estacionalidad marcada y se registró en pocos meses, fenología observada, sobre todo en aquellos sitios donde es más abundante $N$. mexicanus con la cual coexiste, como ocurrió en el área de estudio y en la sierra de Manantlán, Jalisco (Rivera-Cervantes y García-Real, 1998). Aun cuando las abundancias llegan a ser muy similares (Naranjo-López y Navarrete-Heredia, 2011), o por el contrario donde $N$. olidus es más abundante que $N$. mexicanus en sitios ubicados por debajo de 2,000 m (Navarrete-Heredia y QuirozRocha, 2000), se puede observar el desfasamiento temporal. Sin embargo, en los lugares donde $N$. olidus es la única especie del género presente, puede encontrarse hasta en 9 meses del año (Quiroz-Rocha et al., 2008; Reyes, 2001).

Lo anterior confirma lo mencionado por Navarrete-Heredia (2009) en el sentido de que las localidades con 3 o 4 especies de Silphidae tienen un desfasamiento en la fenología de las especies con estrategias similares en la explotación del recurso, probablemente para evitar la competencia, como ocurrió con las 2 especies de Nicrophorus, las cuales se enfocan en cadáveres grandes, además de que algunas especies están representadas por pocos organismos. Por otro lado, las especies que llevan a cabo un aprovechamiento de cadáveres de tallas distintas pueden coexistir, como fue el caso de N.olidus y O.discicolle; esta última pertenece a la subfamilia Silphinae, cuyas especies prefieren aprovechar cadáveres pequeños (Navarrete-Heredia, 2009). Aunque esto al parecer no evita que exista competencia entre ellas, Arellano et al. (2005) mencionan un desplazamiento espacial y temporal en el periodo de actividad de ambas especies en un bosque mesófilo de montaña en Veracruz, en observaciones realizadas durante 5 años consecutivos. Estos autores encontraron que cuando $N$. olidus es muy abundante, las poblaciones de $O$. discicolle disminuyen, y viceversa. En varios estudios se ha visto que ambas especies son más abundantes por debajo de los 2,000 m, y O.discicolle siempre es menos abundante que N. olidus (Méndez, 2002; Quiroz-Rocha et al., 2008; Reyes, 2001; Trevilla-Rebollar et al., 2010), con excepción de lo observado en Gómez Farías, Jalisco, México, donde la especie predominante fue $O$. discicolle (Naranjo-López y Navarrete-Heredia, 2011).

En el caso de Scarabaeidae, de la que se obtuvieron solo 2 especies de Scarabaeinae, se ha observado que la riqueza de esta subfamilia disminuye con el incremento en la altitud en los bosques de pino, pino-encino y mesófilo de montaña, donde se han encontrado entre 4 y 5 especies (Anduaga, 2000; RiveraCervantes y García-Real, 1998), o ninguna en el bosque de pino por arriba de los 3,600 $\mathrm{m}$ de altitud (Cejudo-Espinosa y Deloya, 2005), y en ambientes áridos como el matorral xerófilo se han registrado hasta 2 especies (Jiménez-Sánchez et al., 2013); sin embargo, son más diversos en localidades donde predomina el bosque tropical caducifolio o perennifolio por debajo de 2,000 $\mathrm{m}$ snm, donde se han encontrado entre 11 y 29 especies (Deloya, 1992, 2003, 1996; Deloya y Morón, 1998; Deloya, Ruiz-Lizárraga y Morón, 1987; Gómez, 2005; Halffter et al., 1995; Ix-Balam, Deloya, Ballina-Gómez, Sauri-Duch y CuevasGlory, 2011; Méndez, 2002; Mora-Aguilar y Montes de Oca, 2009; Naranjo-López y Navarrete-Heredia, 2011; Reyes, 2001; Trevilla-Rebollar et al., 2010).

Oniticellus rhinocerulus fue la especie más abundante de Scarabaeidae, la cual tuvo marcada preferencia por las lluvias. 
Esta misma abundancia y fenología fue observada en Manantlán, Jalisco, en un bosque de pino a 2,100 m de altitud (RiveraCervantes y García-Real, 1998); en San José de los Laureles, Morelos, en localidades con bosque mesófilo de montaña alrededor de los 1,800 m (Navarrete-Heredia y Quiroz-Rocha, 2000); en La Michilia, Durango, en bosque de encino-pino por arriba de 2,200 m (Morón y Deloya, 1991), y en Malinalco, Estado de México, en un bosque de pino-encino a 2,300 m (TrevillaRebollar et al., 2010). Se trata de una especie relicta, abundante en los bosques de pino y encino establecidos entre los 1,500 y 2,700 m de altitud (Morón, 2003, citada como Liatongus rhinocerulus). Los adultos han sido recolectados principalmente en sustratos en descomposición como hongos, carroña y excremento de ganado bovino; este último recurso es utilizado para la nidificación (Anduaga y Halffter, 1993; Morón y Deloya, 1991, citada como L. rhinocerulus).

Para O.c. chevrolati solamente se recolectó un ejemplar en junio y otro en julio. Sin embargo, en la sierra de Manantlán, Jalisco, fue la segunda especie de Scarabaeidae más abundante, donde solo estuvo ausente en 3 meses y posiblemente bivoltina (Rivera-Cervantes y García-Real, 1998). Esta tiene una amplia distribución a lo largo del Eje Volcánico Transversal, capturada desde los 2,400 a los 3,800 m snm (Halffter, Verdú, Márquez y Moreno, 2008) en bosques de pino, abeto, pino-encino, encino $\mathrm{y}$ en algunos casos en terrenos desmontados no muy alejados de las áreas boscosas (Zunino y Halffter, 1988).

Se capturaron 2 ejemplares de $X$. thestalus en julio, aunque su presencia en la NTP-80 fue considerada accidental; se sabe que las larvas se alimentan de hojarasca en descomposición (Morón y Zaragoza, 1976), y se desconocen las preferencias alimentarias de los adultos, pero es posible que consuman restos vegetales degradados (Ratcliffe y Morón, 1997). Lo anterior explica su baja abundancia, aun cuando en el área de estudio X. thestalus tuvo poblaciones grandes en el periodo de lluvias, donde se les observó durante el día desplazándose sobre el suelo y volando al atardecer, además de que fueron atraídos de forma abundante a la trampa de luz negra (E. Jiménez-Sánchez, observación personal); estas mismas observaciones fueron hechas para la especie por Morón y Zaragoza (1976) en Villa de Allende, Estado de México. En Gómez Farías, Jalisco, se recolectó un ejemplar de Xyloryctes corniger Bates, 1888, y 2 Xyloryctes howdenorum Delgado y Nájera, 1992, en NTP-80 (Naranjo-López y Navarrete-Heredia, 2011), lo cual reafirma que la captura de las especies del género en este tipo de trampa es accidental, por la actividad de los adultos en el suelo.

De la familia Hybosoridae (sensu Bouchard et al., 2011) se capturó una especie no identificada de Germarostes, género del que se conocen 45 especies, de las cuales 9 han sido registradas para México y ninguna de ellas para el Estado de México (Howden, 2003). Sin embargo, hay una gran cantidad de especies no descritas. De acuerdo con la información que existe sobre la biología de algunas especies del género, no hay evidencias previas de su captura en la trampa permanente tipo NTP-80 en el país, aunque sí existen registros ocasionales de especies de esta familia y de otras subfamilias en trampa cebadas con pescado, calamar y excremento humano (Delgado, Castro-Ramírez, Morón y Ruiz-Montoya, 2012; Favila, 2005; Howden, 2003).
El grupo ha sido considerado saprófago y posiblemente un gran número de especies sean micófagas especializadas (Howden, 2003), por lo que su presencia en la NTP-80 podría ser accidental. Otros métodos de captura para los adultos han sido sacudiendo ramas, tamizando hojarasca y desperdicios de los hormigueros del género Atta; además, son atraídos a trampas de luz o recolectados con trampas de intercepción de vuelo (Howden, 2003).

Ceratotrupes bolivari pertenece al gremio de los coprófagos, y aunque en el presente estudio solo estuvo representada por 4 individuos, esta especie fue capturada abundantemente empleando la NTP-80 en La Michilia, Durango; esto se debe a que los adultos, además de alimentarse de estiércol bovino o equino - el cual buscan para colocar sus huevos-, pueden acudir de manera alternativa a la carroña y a los hongos descompuestos para alimentarse. Además, tiene amplia distribución montana en bosques de pino, encino, encino-pino y en praderas a altitudes de 1,600 a 2,800 m (Howden, 2003; Méndez, 2002; Morón y Deloya, 1991).

De las especies mexicanas de Trox, T.plicatus es la más ampliamente distribuida. Se le ha registrado desde el sur de los Estados Unidos de América hasta el centro-sur de México (Vaurie, 1955); además, se le localiza en bosques de QuercusPinus entre los 2,000 y 2,400 m (Deloya, 2000). En Coatepec Harinas fue rara su presencia en la carroña; sin embargo, Morón y Deloya (1991) la capturaron preferentemente en este mismo recurso, aunque también la recolectaron con la trampa de luz, exclusivamente en el periodo de lluvias. De manera general, la familia Trogidae es más diversa en el matorral xerófilo y el bosque tropical caducifolio (Morón, 2003).

La gran diversidad de especies de plantas presentes en estas cañadas y sus especies epífitas, así como el sotobosque con coberturas del 30 al 50\% y su cercanía con el arroyo, debieron haber propiciado condiciones favorables para la presencia y abundancia de $N$. mexicanus, $N$. olidus, $O$. discicolle y O. rhinocerulus. Para el caso de las 2 primeras especies se ha observado que pueden ver disminuidas sus poblaciones en bosques alterados (Rivera-Cervantes y García-Real, 1998). Por otro lado, la baja abundancia de $X$. thestalus y Germarostes sp. puede explicarse por no tener afinidad hacia la carroña, y en el caso de O.c. chevrolati y T. plicatus posiblemente tienen preferencia por hábitats con diferentes características. La identificación de relaciones estrechas de las especies con alguna característica de su hábitat ha sido utilizada para reconocer especies indicadoras (McGeoch, van Rensburg y Botes, 2002), de tal manera que la composición faunística, la abundancia, la diversidad y la estacionalidad están influenciadas por la complejidad del hábitat, la cual puede estar dada por una serie de variables, entre las que se encuentran la cobertura de los diferentes estratos de vegetación, humedad del suelo, cantidad de hojarasca, troncos, rocas y materia orgánica, los cuales generan microclimas particulares (Lassau, Hochuli, Cassis y Reid, 2005).

La baja abundancia de O.c. chevrolati se debe quizá a que en algunos casos puede habitar en bosques desmontados en la cercanía de zonas boscosas (Zunino y Halffter, 1988). Esta preferencia parece ser común en algunas especies de Onthophagus (McGeoch et al., 2002) y en general de las especies de 
Scarabaeinae de tallas pequeñas, las cuales se ha observado que muestran una mayor asociación con bosques perturbados por el fuego (De Andrade et al., 2014). Sin embargo, en estudios relacionados con escarabajos asociados a cadáveres se encontró que la familia Scarabaeidae - considerando tanto a las especies de tallas grandes como a las pequeñas- fue más abundante en ambientes con sombra que en lugares abiertos, mientras que la familia Trogidae tuvo una respuesta contraria (Caballero y León-Cortés, 2014), esto último coincide con el hecho de que T. plicatus haya sido poco abundante, debido a que tiene preferencia por hábitats con condiciones climáticas más extremas, como el matorral xerófilo (Morón, 2003).

La variación en la abundancia y la riqueza de especies encontrada en este trabajo debe ser interpretada con precaución, y antes de establecer generalizaciones para la fauna de escarabajos necrófilos en el área de cañadas de Coatepec Harinas, es necesario llevar a cabo estudios fuera de estos sitios donde haya una evidente destrucción del hábitat. Asimismo, aunque de manera general la fauna de escarabajos necrófilos de Coatepec Harinas estuvo compuesta por especies de amplia distribución montana, algunas de ellas características del Eje Neovolcánico y la sierra Madre Occidental, es necesario considerar también algunos factores históricos para interpretar las diferencias entre la diversidad y la composición faunística, para que esta información pueda ser empleada en las prácticas de manejo para la conservación.

\section{Agradecimientos}

A Roberto Quezada-García por su asesoría en los análisis estadísticos, a Cuauhtémoc Deloya por la identificación de la especie de Trogidae y a Claudia Arriaga por su apoyo en el trabajo de campo. Así como a 2 revisores anónimos y a Mario E. Favila por sus comentarios que ayudaron a mejorar el escrito. Este estudio fue financiado parcialmente por el Proyecto Zoología de la División de Investigación y Posgrado de la FES-Iztacala, UNAM.

\section{Referencias}

Anduaga, S. (2000). Escarabajos coprófagos (Col. Scarabaeoidea) asociados a hongos en la sierra Madre Occidental, Durango, México: con una compilación de las especies micetófagas. Acta Zoológica Mexicana (nueva serie), 80, 119-130.

Anduaga, S. y Halffter, G. (1993). Nidificación y alimentación de Liatongus rhinocerulus (Bates) (Coleoptera: Scarabaeidae: Scarabaeinae). Acta Zoológica Mexicana (nueva serie), 57, 1-14

Arellano, L., Favila, M. E. y Huerta, C. (2005). Diversity of dung and carrion beetles in a disturbed Mexican tropical montane cloud forest and on shade coffee plantations. Biodiversity and Conservation, 14, 601-615.

Arriaga L., Espinoza J.M., Aguilar C., Martínez E., Gómez L., Loa E. (coordinadores) (2000). Regiones terrestres prioritarias de México. Comisión Nacional para el Conocimiento y Uso de la Biodiversidad. México [consultado 19 Abr 2014]. Disponible en: http://www.conabio.gob.mx/ conocimiento/regionalizacion/doctos/terrestres.html

Bitar, A. y Delgado, L. (2009). A new species of Xyloryctes Hope (Scarabaeidae: Dynastinae) from Guatemala, with a key to the species. The Coleopterists Bulletin, 63, 213-217.
Bouchard, P., Bousquet, Y., Davies, A. E., Alonso-Zarazaga, M. A., Lawrence, J. F., Lyal, C. H. et al. (2011). Family-group names in Coleoptera (Insecta). ZooKeys, 972, 1-972.

Caballero, U. y León-Cortés, J. L. (2012). High diversity beetle assemblages attracted to carrion and dung in threatened tropical oak forests in southern Mexico. Journal of Insect Conservation, 16, 537-547.

Caballero, U. y León-Cortés, J. L. (2014). Beetle succession and diversity between clothed sun-exposed and shaded pig carrion in a tropical dry forest landscape in southern Mexico. Forensic Science International, 245C, $143-150$.

Ceballos G. (comp.) (2011). Propuesta de recategorización y decreto del Parque Nacional Nevado de Toluca. Estado de México, Secretaría de Medio Ambiente y Gobierno del Estado de México, H. Ayuntamiento de Toluca.

Cejudo-Espinosa, E. y Deloya, C. (2005). Coleoptera necrófilos del bosque de Pinus hartwegii del Nevado de Toluca, México. Folia Entomológica Mexicana, 44, 67-73.

Conagua (Comisión Nacional del Agua) (2011). Normales climatológicas por estación. México [consultado 8 Nov 2011]. Disponible en: http://smn.cna.gob.mx/index.php?option=com_content\&view=article\&id= $42 \&$ Ite mid $=28$

Conanp (Comisión Nacional de Áreas Naturales Protegidas) (2013). Estudio previo justificativo para la modificación de la declaratoria del Parque Nacional Nevado de Toluca. Estado de México: Semarnat-Conanp.

De Andrade, R. B., Barlow, J., Louzada, J., Vaz-de Mello, F. Z., Silveira, J. M. y Cochrane, M. A. (2014). Tropical forest fires and biodiversity: Dung beetle community and biomass responses in a northern Brazilian Amazon forest. Journal of Insect Conservation, 18, 1097-1104.

Delgado, L., Pérez, A. y Blackaller, J. (2000). Clave para determinar los taxones genéricos y supragenéricos de Scarabaeoidea Latreille, 1802 (Coleoptera) de México. Folia Entomológica Mexicana, 110, 87-99.

Delgado, J. M., Castro-Ramírez, A. E., Morón, M. A. y Ruiz-Montoya, L. (2012). Diversidad de Scarabaeoidea (Coleoptera) en las principales condiciones de hábitat de Montebello, Chiapas, México. Acta Zoológica Mexicana (nueva serie), 28, 185-210.

Deloya, C. (1992). Necrophilous Scarabaeidae and Trogidae beetles of tropical deciduous forest in Tepexco, Puebla, Mexico. Acta Zoológica Mexicana (nueva serie), 52, 1-11.

Deloya, C. (1996). Los macro-coleópteros necrófilos de Tepoztlán, Morelos, México (Scarabaeidae, Trogidae, Silphidae). Folia Entomológica Mexicana, 97, 9-54.

Deloya, C. (2000). Distribución de la familia Trogidae en México (Coleoptera: Lamellicornia). Acta Zoológica Mexicana (nueva serie), 81, 63-76.

Deloya, C. (2003). Coleoptera Scarabaeidae y Trogidae necrófilos de Valle de Vázquez Los Hornos, Morelos, México. Folia Entomológica Mexicana, 42, 265-272.

Deloya, C. y Morón, M. A. (1994). Coleópteros lamelicornios del distrito de Jojutla, Morelos, México (Melolonthidae, Scarabaeidae, Trogidae y Passalidae). Listados faunísticos de México. Universidad Nacional Autónoma de México, 5, 1-49.

Deloya, C. y Morón, M. A. (1998). Scarabaeoidea (Insecta: Coleoptera) necrófagos de Los Tuxtlas, Veracruz y Puerto Ángel, Oaxaca, México. Dugesiana, $5,17-28$.

Deloya, C., Ruiz-Lizárraga, G. y Morón, M. A. (1987). Análisis de la entomofauna necrófila en la región de Jojutla, Morelos, México. Folia Entomológica Mexicana, 73, 157-171.

Deloya, C., Parra-Tabla, V. y Delfín-González, H. (2007). Fauna de coleópteros Scarabaeidae Laparosticti y Trogidae (Coleoptera: Scarabaeoidea) asociados al bosque mesófilo de montaña, cafetales bajo sombra y comunidades derivadas en el centro de Veracruz, México. Neotropical Entomology, 36, $5-21$.

Favila, M. E. (2005). Diversidad alfa y beta de los escarabajos del estiércol (Scarabaeinae) en Los Tuxtlas, México. En G. Halffter, J. Soberón, P. Koleff, y A. Melic (Eds.), Sobre diversidad biológica: el significado de las diversidades alfa, beta y gamma (pp. 209-219). Zaragoza: Monografías Tercer Milenio, SEA., Conabio, Grupo Diversitas y Conacyt.

García, E. (1973). Modificaciones al sistema de clasificación climática de Köppen (2. ${ }^{a}$ edición). México: Instituto de Geografía. Universidad Nacional Autónoma de México. 
Gómez, G. (2005). Los macro-coleópteros necrófilos (Scarabaeidae, Trogidae y Silphidae) de la Reserva de la Biosfera Sierra de Huautla, Morelos, México. Tesis. Facultad de Estudios Superiores Iztacala, Universidad Nacional Autónoma de México.

Halffter, G. y Martínez, A. (1962). Monografía del género Ceratotrupes Jekel (Col. Scarab. Geotrup.). Ciencia, 21, 145-149.

Halffter, G., Favila, M. E. y Arellano, L. (1995). Spatial distribution of three groups of Coleoptera along an altitudinal transect in the Mexican Transition Zone and its biogeographical implications. Elytron, 9, 151-185.

Halffter, G., Verdú, J. R., Márquez, J. y Moreno, C. (2008). Biogeographical analysis of the Scarabaeinae and Geotrupinae along a transect in central Mexico (Coleoptera, Scarabaeoidea). Fragmenta Entomologica, 40, 273-322.

Hammer, Ø., Harper, D. A. T. y Ryan, P. D. (2001). Past: Paleontological statistics software package for education and data analysis. Paleontología Electrónica, $4,10-50$.

Howden, H. F. (2003). Subfamilia Geotrupinae. En M. A. Morón (Ed.), Atlas de los escarabajos de México, Coleoptera Lamellicornia. Volumen II. Familias Scarabaeidae, Trogidae, Passalidae y Lucanidae (pp. 95-106). Barcelona: Argania Editio.

Ix-Balam, M., Deloya, C., Ballina-Gómez, H., Sauri-Duch, E. y Cuevas-Glory, L. (2011). Diversidad de escarabajos necrófagos (Coleoptera: Scarabaeidae y Trogidae) de la selva baja caducifolia de Conkal, Yucatán, México. En S. Cruz, J. Tello, A. Mendoza, y A. Morales (Eds.), Entomología mexicana (Vol. 10) (pp. 177-181). Texcoco, Estado de México: Sociedad Mexicana de Entomología, A.C.

Jiménez-Sánchez, E., Labrador, G., López, E., Navarrete-Heredia, J. L. y Padilla, J. (2009). Conocimiento actual de los escarabajos (Coleoptera: Staphylinidae, Silphidae, Scarabaeidae y Trogidae) en el Estado de México. En G. Ceballos, R. List, G. Garduño, R. López-Cano, M. J. Muñozcano-Quintanar, y E. Collado, et al. (Eds.), La diversidad biológica del Estado de México, estudio de estado (pp. 97-102). Toluca, Estado de México: Colección Mayor Gobierno del Estado de México.

Jiménez-Sánchez, E., Quezada-García, R. y Padilla-Ramírez, J. (2013). Diversidad de escarabajos necrófilos (Coleoptera: Scarabaeidae, Silphidae, Staphylinidae y Trogidae) en una región semiárida del valle de Zapotitlán de las Salinas, Puebla, México. Revista de Biología Tropical, 61, 1475-1491.

Lassau, S. A., Hochuli, D. F., Cassis, G. y Reid, C. A. M. (2005). Effects of habitat complexity on forest beetle diversity: Do functional groups respond consistently? Diversity and Distributions, 11, 73-82.

Magurran, A. E. (1989). Diversidad ecológica y su medición. Barcelona: Vedra.

McGeoch, M. A., van Rensburg, B. J. y Botes, A. (2002). The verification and application of bioindicators: a case study of dung beetles in a savanna ecosystem. Journal of Applied Ecology, 39, 661-672.

Méndez, R. (2002). Macro-coleópteros necrófilos (Silphidae, Trogidae, Geotrupidae y Scarabaeidae) de la sierra de Nanchititla, Estado de México. Tesis. Estado de México: Facultad de Estudios Superiores Iztacala, Universidad Nacional Autónoma de México.

Mora-Aguilar, E. F. y Montes de Oca, E. (2009). Escarabajos necrófagos (Coleoptera: Scarabaeidae y Trogidae) de la región central baja de Veracruz, México. Acta Zoológica Mexicana (nueva serie), 25, 569-588.

Morón, M. A. (2003). Antecedentes. En M. A. Morón (Ed.), Atlas de los escarabajos de México. Coleoptera: Lamellicornia. Vol. II. Familias Scarabaeidae, Trogidae, Passalidae y Lucanidae (pp. 11-18). Barcelona: Argania Editio.

Morón, M. A. y Deloya, C. (1991). Los coleópteros lamelicornios de la Reserva de la Biosfera La Michilia, Durango, México. Folia Entomológica Mexicana, 81, 209-283.
Morón, M. A. y Terrón, R. (1984). Distribución altitudinal y estacional de los insectos necrófilos en la sierra Norte de Hidalgo, México. Acta Zoológica Mexicana (nueva serie), 5, 1-47.

Morón, M. A. y Zaragoza, S. (1976). Coleópteros Melolonthidae y Scarabaeidae de Villa de Allende, Estado de México. Anales del Instituto de Biología, Universidad Nacional Autónoma de México. Serie Zoológica, 47, 83-118.

Naranjo-López, A. y Navarrete-Heredia, J. L. (2011). Coleópteros necrócolos (Histeridae, Silphidae y Scarabaeidae) en dos localidades de Gómez Farías, Jalisco, México. Revista Colombiana de Entomología, 37, 103-110.

Navarrete-Heredia, J. L. (1995). Coleópteros Silphidae de Jalisco y del Volcán de Tequila incluyendo comentarios generales sobre su biología. Dugesiana, $2,11-26$.

Navarrete-Heredia, J. L. (2009). Silphidae (Coleoptera) de México: diversidad y distribución. Guadalajara, Jalisco: Universidad de Guadalajara.

Navarrete-Heredia, J. L. y Quiroz-Rocha, G. A. (2000). Macro-coleópteros necrófilos de San José de los Laureles, Morelos, México (Coleoptera: Scarabaeidae y Silphidae). Folia Entomológica Mexicana, 110, 1-13.

Peck, S. B. y Anderson, R. S. (1985). Taxonomy, phylogeny and biogeography of the carrion beetles of Latin America (Coleoptera: Silphidae). Quaestiones Entomologicae, 21, 247-317.

Quiroz-Rocha, G. A., Navarrete-Heredia, J. L. y Martínez, P. (2008). Especies de Scarabaeinae (Coleoptera: Scarabaeidae) y Silphidae (Coleoptera) necrófilas de bosque de pino-encino y bosque mesófilo de montaña en el municipio de Mascota, Jalisco, México. Dugesiana, 15, 27-37.

Ratcliffe, B. C. y Morón, M. A. (1997). Dynastinae. En M. A. Morón, B. C. Ratcliffe, y C. Deloya (Eds.), Atlas de escarabajos de México (pp. 53-98), Veracruz: Conabio-Sociedad Mexicana de Entomología, A.C.

Reyes, G. (2001). Los coleópteros saprófagos (Scarabaeidae, Silphidae y Trogidae) del Salto de las Granadas, Guerrero, México. Tesis. Facultad de Estudios Superiores Iztacala, Universidad Nacional Autónoma de México.

Rivera-Cervantes, L. E. y García-Real, E. (1998). Análisis preliminar sobre la composición de los escarabajos necrófilos (Coleoptera: Silphidae y Scarabaeidae) presentes en dos bosques de pino (uno dañado por fuego), en la estación científica Las Joyas, sierra de Manantlán, Jalisco, México. Dugesiana, 5, 11-22.

Terrón, R. A., Anduaga, S. y Morón, M. A. (1991). Análisis de la coleopterofauna necrófila de la Reserva de la Biosfera La Michilia, Durango, México. Folia Entomológica Mexicana, 81, 315-324.

Toledo, T. (2009). El bosque de niebla. Biodiversitas, 83, 1-6.

Torres-Zúñiga, M. y Tejero-Diez, D. (1998). Flora y vegetación de la sierra de Sultepec, Estado de México. Anales del Instituto de Biología, Universidad Nacional Autónoma de México. Serie Botánica, 69, 135-174.

Trevilla-Rebollar, A., Deloya, C. y Padilla-Ramírez, J. (2010). Coleópteros necrófilos (Scarabaeidae, Silphidae y Trogidae) de Malinalco, Estado de México, México. Neotropical Entomology, 39, 486-495.

Vaurie, P. (1955). A revision of the genus Trox in North America (Coleoptera, Scarabaeidae). Bulletin of the American Museum of Natural History, 106, $1-89$.

Zar, J. H. (1999). Biostatistical analysis (4th edition). New Jersey: Prentice Hall

Zaragoza, S. y Pérez, H. (1975). Varianza de Nicrophorus mexicanus Matt. (Coleoptera: Silphidae) y su correlación ambiental en El Pedregal de San Ángel, Distrito Federal, México. Anales del Instituto de Biología, Universidad Nacional Autónoma de México. Serie Zoología, 1, 459-475.

Zunino, M. y Halffter, G. (1988). Análisis taxonómico, ecológico y biogeográfico de un grupo americano de Onthophagus (Coleoptera: Scarabaeidae). Monografía IX. Torino: Museo Regionale di Scienze Naturali. 